\title{
Role of heat source/sink on time dependent free convective flow in a coaxial cylinder filled with porous material: a semi analytical approach
}

Taiwo S. Yusuf, Gambo Dauda

Department of Mathematics, Ahmadu Bello University, Nigeria

\begin{tabular}{l}
\hline \hline Article Info \\
\hline Article history: \\
Received Apr 3, 2019 \\
Revised Sep 13, 2019 \\
Accepted Feb 19, 2020
\end{tabular}

\section{Keywords:}

Annulus

Free convection

Heat source/sink

Riemann-sum approximation

Unsteady

\begin{abstract}
In this article, the semi analytical solution for a fully developed time dependent free convective flow of a viscous incompressible fluid with heat source/sink in an infinite vertical coaxial cylinder saturated with porous material has been analyzed. The flow was induced by buoyancy forces due to temperature differences caused by the thermal insulation of the inner wall and constant heating of the outer wall. The Laplace transform technique was employed to transform the governing equation from time domain to the Laplace domain. Notwithstanding, a numerical inversing scheme known as Riemann-sum approximation (RSA), renowned for its precision has been utilized to transform the Laplace domain solution to time domain. The accuracy of the numerical technique employed was tested by presenting a comparison with the numerical values obtained using RSA, PDEPE, and steady state solution at large time. The effects of the various flow parameters on the flow formation are exhibited graphically. It is interesting to note that the fluid temperature and velocity increases as time passes. In addition, the velocity can be enhanced and minimized by gradually increasing Darcy number and the viscosity ratio respectively. However, the increase is seen to be more prominent when heat source is applied. The drag on both walls are seen to increase with increase in Darcy number, the reverse trend is observed with increase in the viscosity ratio.
\end{abstract}

This is an open access article under the CC BY-SA license.

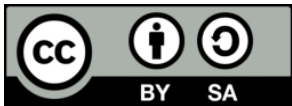

\section{Corresponding Author:}

Taiwo S. Yusuf,

Department of Mathematics,

Ahmadu Bello University,

Community Market, Zaira Nigeria, local 810211, Zaria, Nigeria.

Email: taiyeee@yahoo.com

\section{NOMENCLATURE}

a Radius of the inner cylinder (m)

$b \quad$ Radius of the outer cylinder (m)

$C_{p} \quad$ Specific heat at constant pressure $(\mathrm{kJ} / \mathrm{kgK})$

Da Darcy number

$g \quad$ Gravitational acceleration $\left(\mathrm{m} / \mathrm{s}^{2}\right)$

$k \quad$ Thermal conductivity of the fluid $(\mathrm{W} / \mathrm{mK})$

$K \quad$ Permeability of the Porous medium $\left(\mathrm{m}^{2}\right)$

$\mathrm{Nu} \quad$ Dimensionless nusselt number

$\operatorname{Pr} \quad$ Prandtl number $(\mu \mathrm{cp} / \mathrm{k})$

$Q_{0} \quad$ Dimensional heat source/sink parameter 

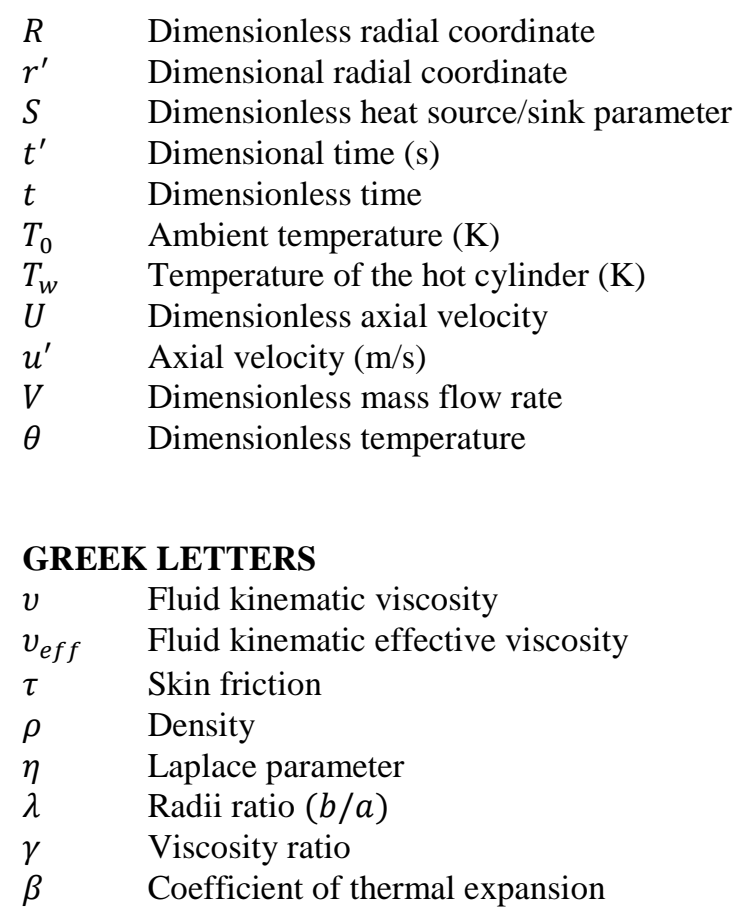

\section{INTRODUCTION}

The study of time dependent free convective flow of viscous incompressible fluid in cylindrical geometry has attracted significant attention by many researchers in the past decades due to its practical applications in the modern industry and engineering such as in nuclear power plants, heating and cooling of chambers, heat exchangers, radiators, electronic cooling and air condition system. Consequently, the problems of free convective flow in cylindrical geometry has been solved by many researchers using both the analytical and numerical approach due to the complexity of the geometry and most importantly when the convection current is set up as a result of the internal heat source/sink in addition to the simultaneous heating and insulation of the walls. Several work has been devoted to examine the role of heat source/sink on convective flows in channel, microchannel and annulus [1-7].

Unsteady free convective flow of heat generating/absorbing fluid in an annulus with permeable walls was semi analytically studied by [1]. They concluded that that the effect of heat generation/absorption can be controlled by increasing/decreasing the viscosity ratio. Subsequently, reference [2] examined laminar unsteady fully developed free convective flow of viscous, incompressible and heat generating/absorbing fluid in an annulus saturated with porous material where the inner cylinder is heated constantly while the outer cylinder is maintained at constant temperature. They reported that in addition to the results obtained from their previous work [1], the heat generating fluid is desirable for optimum mass flux in the annular gap most importantly when the convection current is enhanced by constant heat flux. The analytical examination for laminar fully developed flow of viscous incompressible and electrically conducting fluid in an annulus in which the wall of the inner cylinder is heated or cooled either isothermally or at a constant heat flux while the outer cylinder is maintained at ambient temperature was considered by [3].

Closed forms solutions for transient fully developed free convection corresponding to four fundamental thermal boundary conditions in vertical concentric annulus was presented by [4-5] reported the analytical solution of a laminar fully developed free convection in between coaxial cylinders partially filled with porous material. Considerable research work has been dedicated to examine the role of heat source/sink on viscous flow for different physical phenomenon. In many of such problems, there may be plausible temperature difference between the surface and the surrounding fluid. This demands the consideration of temperature dependent heat source/sink which may exert strong effect on the heat transfer characteristics. An analytical solution for the combined effect of heat source, porosity and thermal radiation on mixed convection flow in a vertical annulus was presented by [6]. His findings illustrated that increase in the radiation parameter and heat source parameter causes an increase in the fluid temperature and rate of heat transfer. Recently, reference [7] analyzed the unsteady flow of a viscous, incompressible, electrically and thermally conducting fluid between two infinite parallel porous walls placed at $y=0$ and $y=a$. 
It is assumed that the electrically conducting fluid is driven by mutual action of the imposed pressure gradient, thermal buoyancy and heat source or sink, other related problems can be found in [8-13].

The effect of buoyant forces on magnetohydrodynamic (MHD) free convective flow of an electrically conducting fluid in the presence of heat source/sink was numerically studied by [14-15] examined the effects of heat source/sink on MHD flow and heat transfer over a shrinking sheet with mass suction. Reference [16] investigated the effects of induced magnetic field and heat source/sink on fully developed laminar natural convective flow of a viscous incompressible and electrically conducting fluid in the presence of radial magnetic field by considering induced magnetic field into account and reported some interesting result, they obtained that increasing value of the heat source/sink parameter leads to increase in velocity. Reference [17] conducted a study on MHD natural convective flow of heat generating/absorbing fluid with slip effect in an annular porous medium. They adopted the Adomian decomposition method (ADM), a numerical scheme to obtain the solution of the governing equation. In the result they presented, heat generation parameter enhanced the temperature and velocity of the fluid in the annular gap. Moreover, slip effect parameter increases the velocity of the fluid.

Employing a numerical shooting technique with a fourth-fifth order Runge-Kutta scheme, reference [18] carried out an investigation to study a problem of the chemical reaction and heat generation or absorption effects on MHD mixed convective boundary layer flow of a nanofluid through a porous medium due to an exponentially stretching sheet. It was found that Nusselt number is a decreasing function of the heat generation/absorption parameter and the chemical reaction parameter. Later on, Reference [19] examined the combined effects of magnetic field and heat generation/absorption on unsteady boundary-layer convective heat and mass transfer of a non-Newtonian nanofluid over a permeable stretching wall and concluded that the thermal and concentration boundary-layer thickness has higher values with the increasing of magnetic field and heat generation in the case of a pseudoplastic nanofluid than others. Other related articles by different researchers addressing some remarkable problems on the effect of heat generation/absorption for different fluids can be seen in [20-25]. The present paper investigates the effect of the heat source/sink presence on time dependent free convective flow in a vertical coaxial cylinder when the fluid is filled with porous material. Retardation due to Lorentz drag force opposing fluid flow is negligible which is therefore neglected. In addition, for effective utilization of the available heat supplied to the system, the inner cylinder is insulated against heat this is to retain heat within the system.

\section{MATHEMATICAL FORMULATION}

Consider a fully developed time dependent free convective flow of a viscous incompressible fluid in the presence of heat source/sink within a vertical coaxial cylinder of infinite length. The axis of cylinder is taken vertically upward in the direction opposite the gravitational force along the $z^{\prime}$-axis, while $r$-axis is taken in the radial direction. The convective current is as a result of the constant heating at the outer cylinder as well as heat source/sink. The radii of inner and outer cylinder are denoted by $a$ and $b$ respectively as depicted in Figure 1. Initially, it is assumed that at time $t^{\prime} \leq 0$ the fluid and the two cylinders at ambient temperature $T_{0}$. At $t^{\prime}>0$, the outer cylinder is assumed to be heated to a temperature $T_{w}$ greater than that of the surrounding fluid and the inner cylinder which is thermally insulated. The flow is thereby setup by the buoyancy force due to the temperature difference of the fluid in the coaxial cylinder. Thus, under the usual Boussinesq approximation, the dimensionless form of the governing equations for the model under consideration can be written as (1-2).

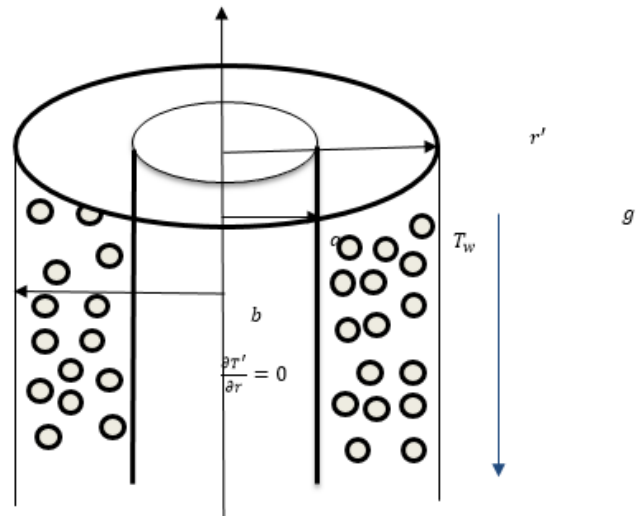

Figure 1. Schematic diagram of the problem 


$$
\begin{aligned}
& \frac{\partial U}{\partial t}=\Gamma\left[\frac{\partial^{2} U}{\partial R^{2}}+\frac{1}{R} \frac{\partial U}{\partial R}\right]-\frac{U}{D a}+\theta \\
& \frac{\partial \theta}{\partial t}=\frac{1}{P r}\left[\frac{\partial^{2} \theta}{\partial R^{2}}+\frac{1}{R} \frac{\partial \theta}{\partial R}\right]+\frac{S \theta}{P r}
\end{aligned}
$$

The $(1,2)$ have been rendered dimensionless using the following non-dimensional quantities:

$$
\begin{aligned}
& t=\frac{t^{\prime} v}{a^{2}}, \quad R=\frac{r^{\prime}}{a} \lambda=\frac{b}{a}, \quad \theta=\frac{\left(T^{\prime}-T_{0}\right)}{T_{w}-T_{0}}, \quad \operatorname{Pr}=\frac{\mu c_{p}}{k} \\
& \Gamma=\frac{V_{\text {eff }}}{v}, \quad D a=\frac{K}{a^{2}}, \quad U=\frac{U \prime}{U_{0}}, \quad U_{0}=\frac{g \beta\left(T w-T_{0}\right) a^{2}}{v}, S=\frac{Q_{0} a^{2}}{k}
\end{aligned}
$$

while the initial and boundary conditions in dimensionless form are given as:

$$
\begin{aligned}
& t \leq 0, U=\theta=0,1 \leq R \leq \lambda \\
& t>0:\left\{\begin{array}{l}
U=0, \frac{\partial \theta}{\partial R}=0 \text { at } R=1 \\
U=0, \theta=1 \text { at } R=\lambda
\end{array}\right.
\end{aligned}
$$

Using $\bar{U}(R, \eta)=\int_{0}^{\infty} U(R, t) e^{-\eta t} d t$ and $\bar{\theta}(R, \eta)=\int_{0}^{\infty} \theta(R, t) e^{-\eta t} d t$ where $\eta$ is the Laplace parameter and $\eta>0$. The Laplace transforms of (1) and (2) are given as:

$$
\begin{aligned}
& \frac{d^{2} \bar{U}}{d R^{2}}+\frac{1}{R} \frac{d \bar{U}}{d R}-\frac{1}{\Gamma}\left[\frac{1}{D a}+\eta\right] \bar{U}+\frac{\bar{\theta}}{\Gamma}=0 \\
& \frac{d^{2} \bar{\theta}}{d R^{2}}+\frac{1}{R} \frac{d \bar{\theta}}{d R}-[\eta \operatorname{Pr}-S] \bar{\theta}=0
\end{aligned}
$$

The Laplace transform of the boundary conditions are given by

$$
\begin{aligned}
& \bar{U}=0 ; \frac{d \bar{\theta}}{d R}=0 \text { at } R=1 \\
& \bar{U}=0 ; \bar{\theta}=\frac{1}{\eta} \text { at } R=\lambda
\end{aligned}
$$

The solutions of the Bessel ordinary differential in $(6,7)$ in the Laplace domain subject to the boundary conditions $(8,9)$ are given:

$$
\begin{aligned}
& \bar{\theta}(R, \eta)=C_{1} I_{0}(R \delta)+C_{2} K_{0}(R \delta) \\
& \bar{U}(R, \eta)=D_{1} I_{0}(R \alpha)+D_{2} K_{0}(R \alpha)-\frac{1}{\Gamma}\left[\frac{C_{1} I_{0}(R \delta)+C_{2} K_{0}(R \delta)}{\left(\delta^{2}-\alpha^{2}\right)}\right]
\end{aligned}
$$

where

$$
\begin{array}{ll}
\delta & =\sqrt{\eta P r-S}, \quad \alpha=\left[\frac{1}{\Gamma}\left(\frac{1}{D a}+\eta\right)\right]^{1 / 2}, \quad C_{1}=\frac{K_{1}(\delta)}{\eta\left[I_{1}(\delta) K_{0}(\lambda \delta)+I_{0}(\lambda \delta) K_{1}(\delta)\right]}, \\
C_{2} & =\frac{I_{1}(\delta)}{\eta\left[I_{1}(\delta) K_{0}(\lambda \delta)+I_{0}(\lambda \delta) K_{1}(\delta)\right]}, \\
D_{2} & =\frac{M_{1} I_{0}(\lambda \alpha)-M_{2} I_{0}(\alpha)}{I_{0}(\lambda \alpha) K_{0}(\alpha)-I_{0}(\alpha) K_{0}(\lambda \alpha)} .
\end{array}
$$

\subsection{Skin frictions, Nusselt number and mass flux}

Using (11), the skin friction at the surface of the cylinders in the Laplace domain at $R=1$ and $R=\lambda$ are given respectively as follows:

$$
\bar{\tau}_{1}(1, \eta)=\left.\frac{d \bar{U}}{d R}\right|_{R=1}=\alpha\left\{D_{1} I_{1}(\alpha)-D_{2} K_{1}(\alpha)\right\}-\frac{\delta}{\Gamma} \frac{\left[C_{1} I_{1}(\delta)-C_{2} K_{1}(\delta)\right]}{\left(\delta^{2}-\alpha^{2}\right)}
$$




$$
\bar{\tau}_{\lambda}(\lambda, \eta)=-\left.\frac{d \bar{U}}{d R}\right|_{R=\lambda}=-\alpha\left\{D_{1} I_{1}(\lambda \alpha)-D_{2} K_{1}(\lambda \alpha)\right\}-\frac{\delta}{\Gamma} \frac{\left[C_{1} I_{1}(\lambda \delta)-C_{2} K_{1}(\lambda \delta)\right]}{\left(\delta^{2}-\alpha^{2}\right)}
$$

Using (10) the rate of heat transfer at the surface of the outer cylinder in the Laplace domain is given by:

$$
\overline{N u}_{\lambda}=-\left.\frac{d \bar{\theta}}{d R}\right|_{R=\lambda}=-\delta\left[\left[C_{1} I_{1}(\lambda \delta)-C_{2} K_{1}(\lambda \delta)\right]\right.
$$
is given by:

Thus, the solution of the mass flow rate in the Laplace domain through the annular region

$$
\bar{V}=2 \pi \int_{1}^{\lambda} R \bar{U} d R=2 \pi\left(E_{1}-E_{2}\right)
$$

where

$$
\begin{aligned}
E_{1} & =\frac{D_{1}}{\alpha}\left(\lambda I_{1}(\lambda \alpha)-I_{1}(\alpha)\right)-\frac{D_{2}}{\alpha}\left(\lambda K_{1}(\lambda \alpha)-K_{1}(\alpha)\right), \\
E_{2} & =\frac{1}{\delta \Gamma\left(\delta^{2}-\alpha^{2}\right)}\left[C_{1}\left(\lambda I_{1}(\lambda \delta)-I_{1}(\delta)\right)-C_{2}\left(\lambda K_{1}(\lambda \delta)-K_{1}(\delta)\right)\right]
\end{aligned}
$$

It is paramount to note that the given solutions above are in Laplace domain. (10-15) is to be transformed to the time domain. Due to the complex nature of these solutions, the Riemann-sum approximation approach was adopted. Following the work of [1] any function in the Laplace domain can be inverted to the time domain as follows:

$$
Z(R, t)=\frac{e^{\eta t}}{t}\left[\frac{1}{2} \bar{Z}(R, \eta)+R e\left(\sum_{n=1}^{M} \bar{Z}\left(R, \varepsilon+\frac{i n \pi}{t}\right)(-1)^{n}\right]\right.
$$

where $Z$ represents $\theta, U, V$ or $N u$ as the case may be, Re refers to the real part of the summation, $i=\sqrt{-1}, M$ is the number of terms used in the Riemann-sum approximation and $\varepsilon$ is the real part of the Bromwich contour that is used in inverting Laplace transforms. The Riemann-sum approximation for the Laplace inversion involves a single summation for the numerical process its accuracy depends on the value of $\varepsilon$ and the truncation error dictated by $M$. According to [26], the value of $\varepsilon t$ that best satisfied the result is 4.7 .

\subsection{Validation of the method}

To validate the numerical scheme adopted in inverting (10-15) in the time domain, we set out to obtain the steady state solution of $(1,2)$, this is achieved by computing the steady state temperature and velocity field analytically which is expected to coincide with transient state solution at large time. This can be done by taking $\frac{\partial()}{\partial t}=0$ in the non-dimensional $(1,2)$ which then reduces to the ordinary differential equations given by:

$$
\begin{aligned}
& \Gamma\left[\frac{d^{2} U}{d R^{2}}+\frac{1}{R} \frac{d U}{d R}\right]-\frac{U}{D a}+\theta=0 \\
& \frac{d^{2} \theta}{d R^{2}}+\frac{1}{R} \frac{d \theta}{d R}+S \theta=0
\end{aligned}
$$

with the given initial and boundary conditions

$$
\begin{aligned}
& U=0 ; \frac{d \theta}{d R}=0 \text { at } R=1 \\
& U=0 ; \theta=1 \text { at } R=\lambda
\end{aligned}
$$

The $(17,18)$ are solved under the boundary conditions $(19,20)$ to obtain the solution of the steady state temperature field, velocity field, skin friction on the surface of the cylinders, Nusselt number and mass flow rate. The solutions are given as:

$$
\theta(R)=A_{1} I_{0}(R \xi)+A_{2} K_{0}(R \xi)
$$




$$
\begin{aligned}
& U(R)=B_{1} I_{0}(R \varphi)+B_{2} K_{0}(R \varphi)+B_{3} I_{0}(R \xi)+B_{4} K_{0}(R \xi) \\
& \tau_{1}=\left.\frac{d U}{d R}\right|_{R=1}=\varphi\left\{B_{1} I_{1}(\varphi)-B_{2} K_{1}(\varphi)\right\}+\xi\left\{B_{3} I_{1}(\xi)-B_{4} K_{1}(\xi)\right\} \\
& \tau_{\lambda}=-\left.\frac{d U}{d R}\right|_{R=\lambda}=-\left[\varphi\left\{B_{1} I_{1}(\lambda \varphi)-B_{2} K_{1}(\lambda \varphi)\right\}+\xi\left\{B_{3} I_{1}(\lambda \xi)-B_{4} K_{1}(\lambda \xi)\right\}\right] \\
& N u_{\lambda}=-\left.\frac{d \theta}{d R}\right|_{R=\lambda}=-\left[\xi\left\{A_{1} I_{1}(\lambda \xi)-A_{2} K_{1}(\lambda \xi)\right\}\right] \\
& V=2 \pi \int_{1}^{\lambda} R U d R=2 \pi\left[B_{5}+B_{6}\right]
\end{aligned}
$$

where

$$
\begin{aligned}
& \xi=\sqrt{-S}, \quad \varphi=\sqrt{\frac{1}{\Gamma D a}}, \quad A_{1}=\frac{K_{1}(\xi)}{I_{1}(\xi) K_{0}(\lambda \xi)+I_{0}(\lambda \xi) K_{1}(\xi)}, \quad A_{2}=\frac{I_{1}(\xi)}{I_{1}(\xi) K_{0}(\lambda \xi)+I_{0}(\lambda \xi) K_{1}(\xi)}, \\
& B_{1}=\frac{M_{4} K_{0}(\varphi)-M_{3} K_{0}(\lambda \varphi)}{I_{0}(\lambda \varphi) K_{0}(\varphi)-I_{0}(\varphi) K_{0}(\lambda \varphi)}, \quad B_{2}=\frac{M_{3} I_{0}(\lambda \varphi)-M_{4} I_{0}(\varphi)}{I_{0}(\lambda \varphi) K_{0}(\varphi)-I_{0}(\varphi) K_{0}(\lambda \varphi)^{\prime}}, \quad B_{3}=\frac{-D a A_{1}}{\xi^{2} \Gamma D a-1}, \quad B_{4}=\frac{-D a A_{2}}{\xi^{2} \Gamma D a-1}, \\
& B_{5}=\frac{B_{1}}{\varphi}\left\{\lambda I_{1}(\lambda \varphi)-I_{1}(\varphi)\right\}-\frac{B_{2}}{\varphi}\left\{\left(\lambda K_{1}(\lambda \varphi)-K_{1}(\varphi)\right\}, B_{6}=\frac{B_{3}}{\xi}\left\{\lambda I_{1}(\lambda \xi)-I_{1}(\xi)\right\}-\frac{B_{4}}{\xi}\left\{\lambda K_{1}(\lambda \xi)-K_{1}(\xi)\right\}\right.
\end{aligned}
$$

To further establish the accuracy of the Riemann-sum approximation approach, the PDEPE is used to solve $(1,2)$ with $(4,5)$ as the initial and boundary condition respectively. The numerical values obtained from the Riemann-Sum approximation, steady state solution and the Parabolic and Elliptic Partial Differential Equations (PDEPE) are presented in Table 1.

Table 1. Numerical values of the transient state velocity obtained using the Riemann-sum approximation method, PDEPE and that obtained from the exact solution

\begin{tabular}{ccccc}
\hline $\mathrm{Da}$ & $\mathrm{T}$ & Riemann-sum approximation & PDEPE & Exact solution \\
\hline 0.1 & 0.2 & 0.0352 & 0.0353 & 0.0608 \\
& 0.4 & 0.0508 & 0.0509 & 0.0608 \\
& 0.6 & 0.0571 & 0.0570 & 0.0608 \\
& Steady state & 0.0608 & 0.0608 & 0.0608 \\
1.0 & 0.2 & 0.0541 & 0.0540 & 0.1128 \\
& 0.4 & 0.0884 & 0.0886 & 0.1128 \\
& 0.6 & 0.1031 & 0.1032 & 0.1128 \\
& Steady state & 0.1128 & 0.1128 & 0.1128 \\
\hline
\end{tabular}

\section{RESULTS AND DISCUSSION}

In order to understand the effects of the various controlling parameters on the fluid temperature, fluid velocity, skin friction on the walls of the cylinder, Nusselt number and mass flow rate in the annular region, a MATLAB program is written to compute and generate the line graphs and numerical values for some selected values of the dimensionless parameter. The present parametric study has been performed over a reasonable range $0.001 \leq D a \leq 1.0,0.5 \leq \Gamma \leq 1.5,-2 \leq S \leq 2$ where $\lambda=2$ is also taken as reference point. Two values of Prandtl number $(P r=0.71)$ and $(P r=7.0)$ were considered all through this work which corresponds to air and water respectively. In the course of this research work, positive values of $S$ denote heat source and is specified in Figures 2-9 (a), while negatives values of $S$ denote heat sink and is specified in figures. 2-9 $(b)$.

Figure 2 shows the temperature profiles for different values of $t$ across the annular gap for both cases of heat source and sink. It is clear from Figure 2 that the fluid temperature increases and attain steady state faster as time increases for both heat source and sink and drops towards the insulated wall with the exception of $\operatorname{Pr}=0.71$ in (a). Generally higher temperature profiles are perceived with decrease in Prandtl number $(\mathrm{Pr})$. Velocity profiles for heat source and sink for various dimensionless parameters are presented in Figures 3-5. It can be seen clearly from Figures 3 and 4, that as time and Darcy number $(D a)$ increases respectively, the fluid velocity is accelerated due to the fact that hot fluid particles enhance convective current, though the increment is significant along the heated wall and most importantly with heat source.

Figure 5 presents the effect of the fluid viscosity ratio $(\Gamma)$ on the velocity It is evident that the effect of viscosity ratio is to retard fluid velocity. It is seen that as the fluid viscosity. Variation of skin friction $\left(\tau_{1}\right)$ 
along the insulated wall are revealed in Figures 6 and 7 with respect to Darcy number $(D a)$ and viscosity ratio $(\Gamma)$ for both heat source and sink. It is found from these figures that the skin friction $\left(\tau_{1}\right)$ along the wall of the inner cylinder increases and drops as Darcy number $(D a)$ and viscosity ratio $(\Gamma)$ of the fluid increases respectively for both heat source and sink. However, the magnitude of the skin friction $\left(\tau_{1}\right)$ is noticeable for the case of heat source. Figures 8 and 9 reveals the variation of skin friction $\left(\tau_{\lambda}\right)$ for different values of Darcy number $(D a)$ and viscosity ratio $(\Gamma)$ along the heated wall for both heat source and sink. It is obvious from the figures that skin friction coefficient $\left(\tau_{\lambda}\right)$ along the outer cylinder is enhanced with increase in Darcy number $(D a)$ and decreases with increase in both Prandtl number $(P r)$ and viscosity ratio $(\Gamma)$. However, an increase in skin friction on both walls is obtained with time. It is interesting to note that skin friction on both walls attain steady state faster in the case of the heat sink. It is good to also note that similar to the observation in Figures 6 and 7. Tables 2 and 3 reveals the numerical values for the rate of heat transfer along the heated wall and the quantity of fluid flowing in the annular gap respectively. It is seen that higher values are perceived as time is increased and heat source/sink parameter is increased that both Nusselt number and mass flow.

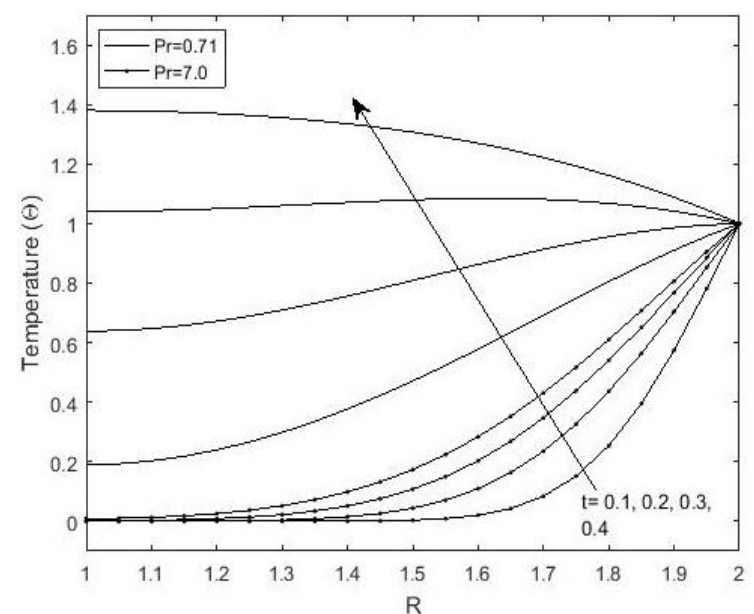

(a)

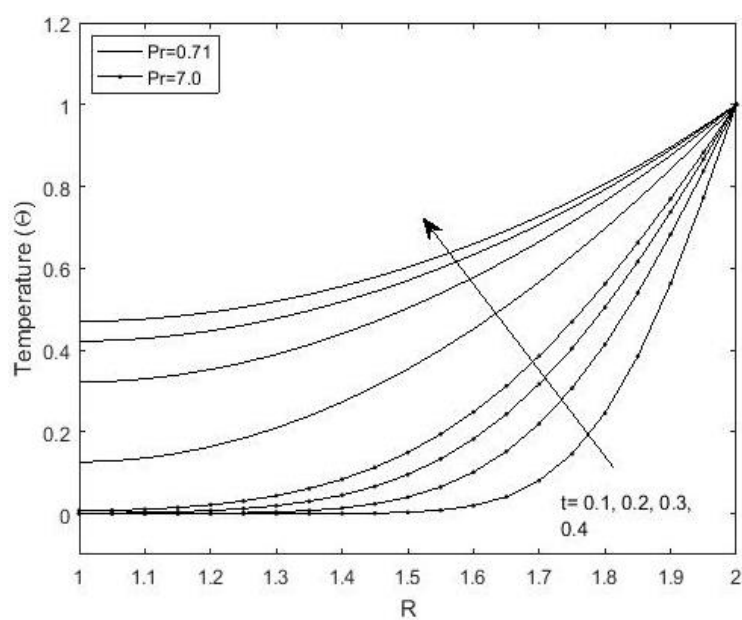

(b)

Figure 2. Temperature profile for different values of $t(S=2.0)$,

(a) Heat source, (b) Heat sink

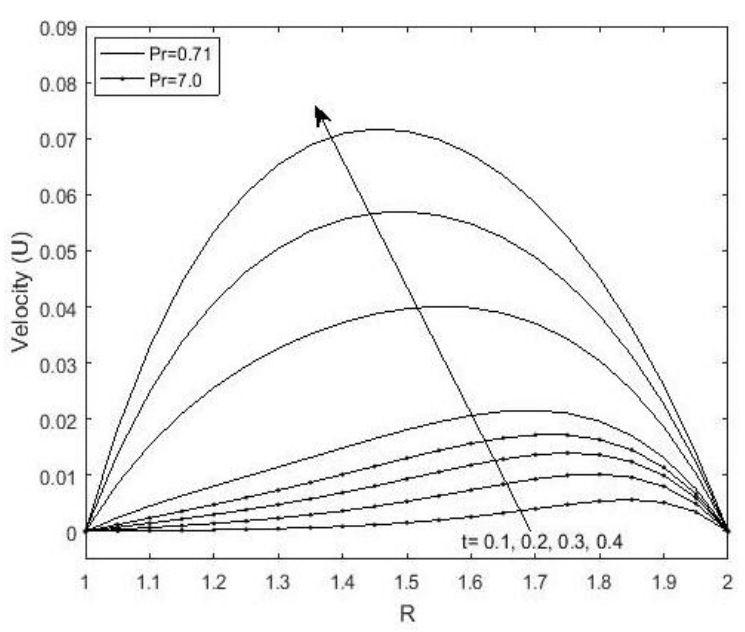

(a)

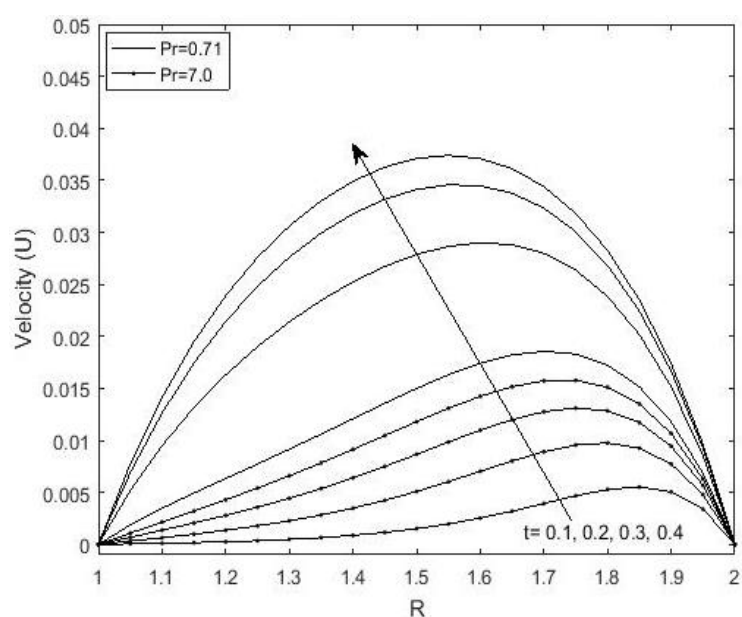

(b)

Figure 3. Velocity profile for different values of $t(S=2.0, D a=0.1, \Gamma=1.0)$,

(a) Heat source, (b) Heat sink 


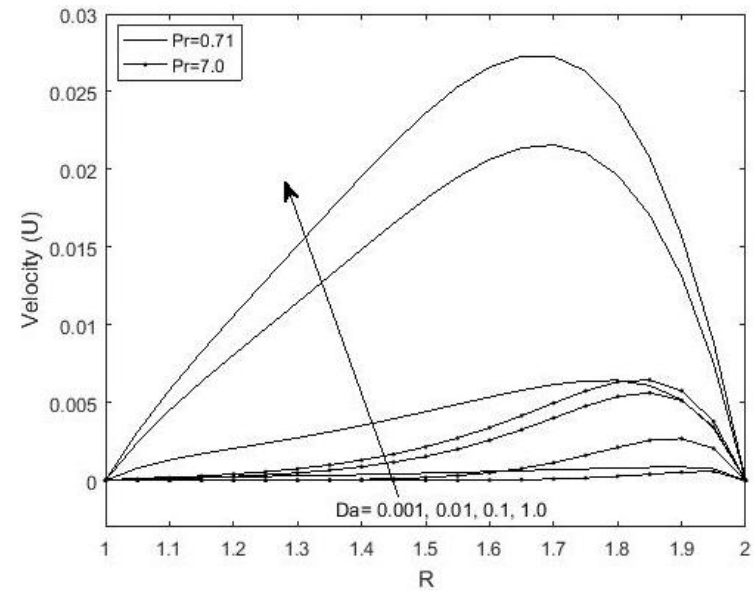

(a)

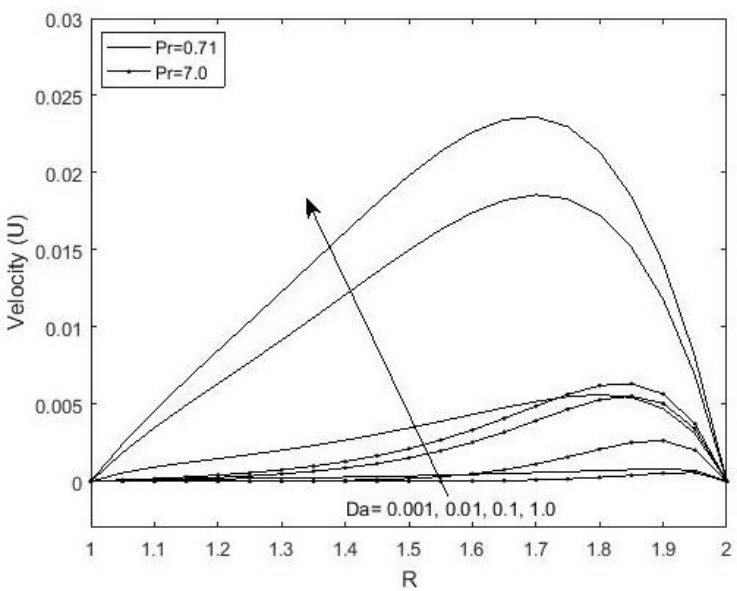

(b)

Figure 4. Velocity profile for different values of $D a(S=2.0, t=0.1, \Gamma=1.0)$, (a) Heat source, (b) Heat sink

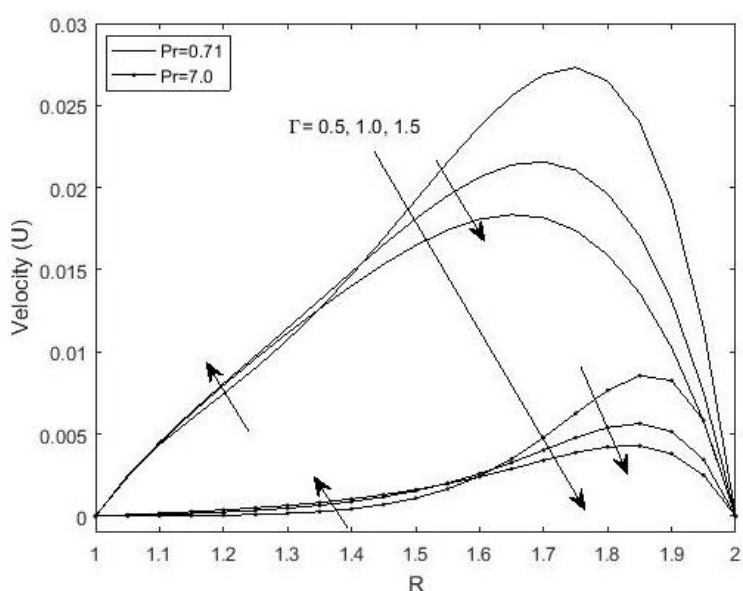

(a)

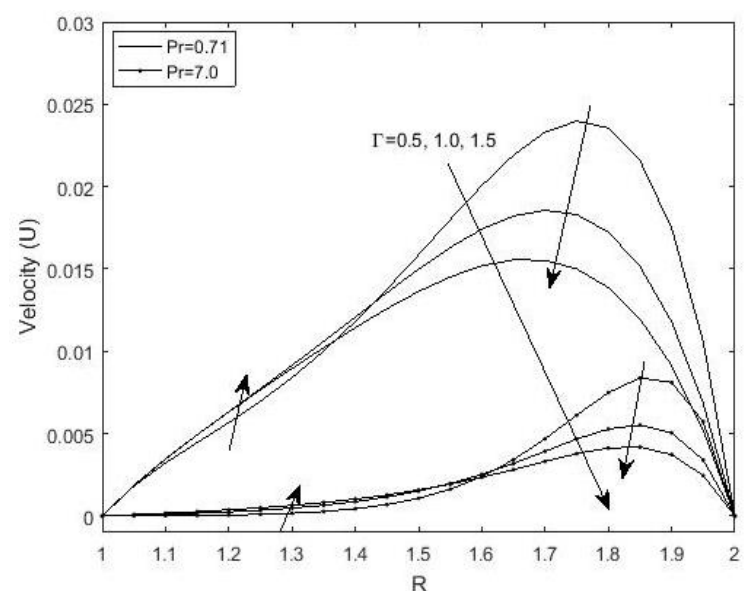

(b)

Figure 5. Velocity profile for different values $\Gamma(S=2.0, t=0.1, D a=0.1)$, (a) Heat source, (b) Heat sink

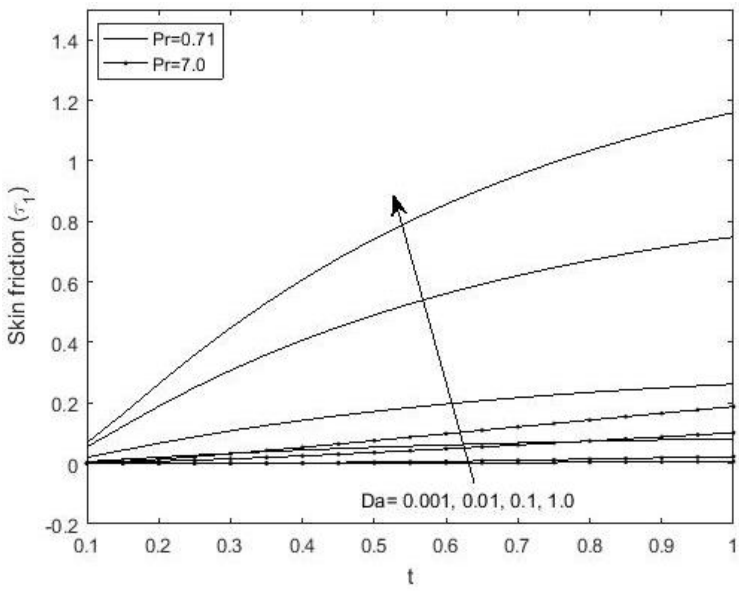

(a)

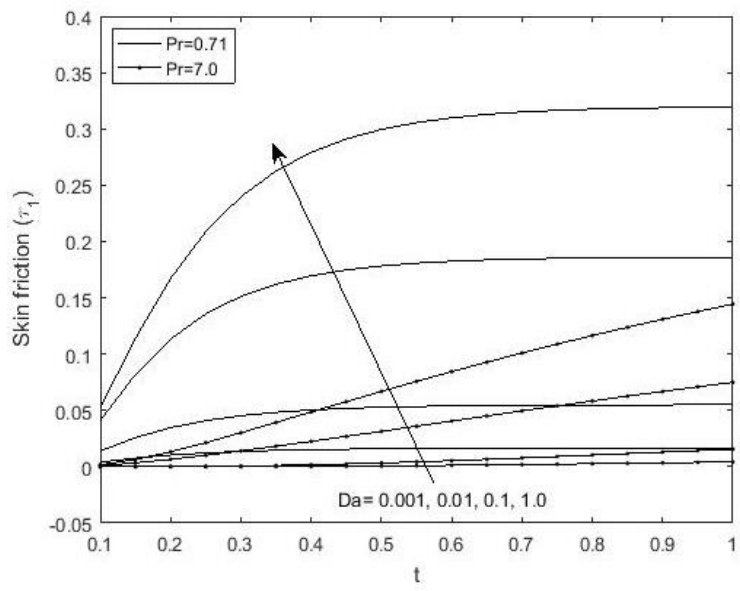

(b)

Figure 6. Variation of skin friction $\left(\tau_{1}\right)$ for different values of $D a(S=2.0, \Gamma=1.0)$,

(a) Heat source, (b) Heat sink 


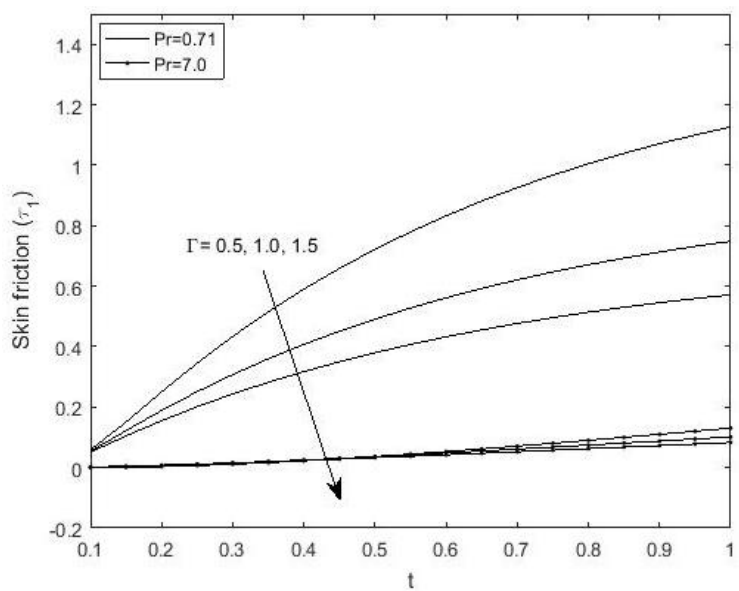

(a)

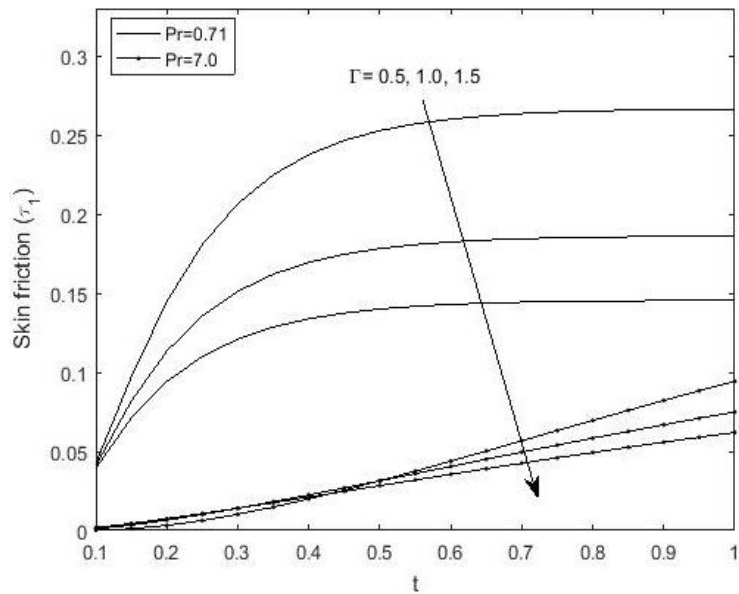

(b)

Figure 7.Variation of skin friction $\left(\tau_{1}\right)$ for different values of $\Gamma(S=2.0, D a=0.1)$, (a) Heat source, (b) Heat sink

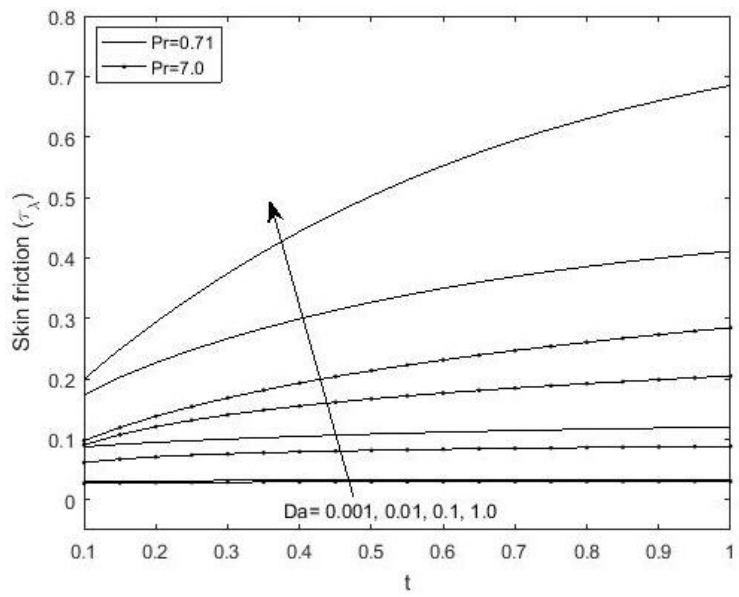

(a)

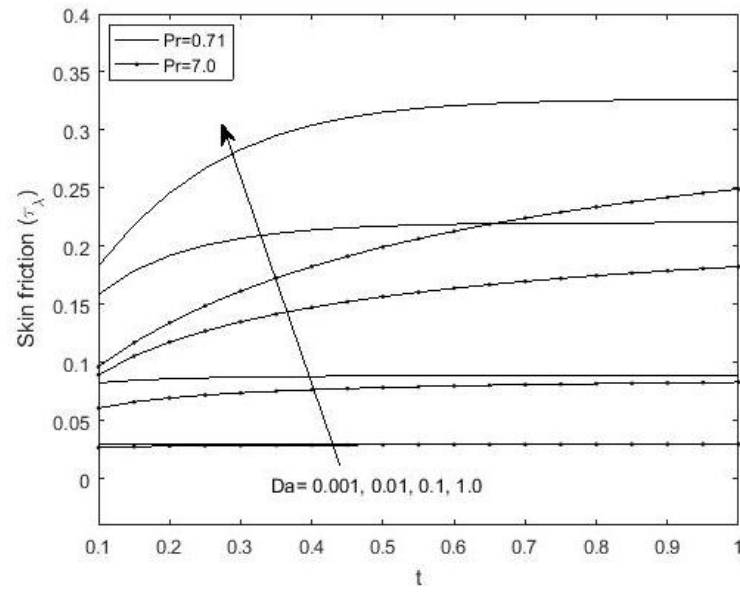

(b)

Figure 8. Variation of skin friction $\left(\tau_{\lambda}\right)$ for different values of $D a(S=2.0, \Gamma=1.0)$, (a) Heat source, (b) Heat sink

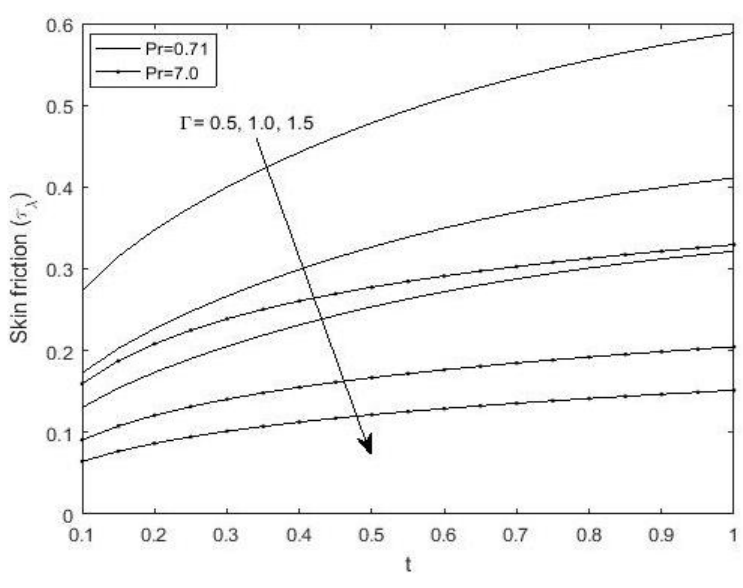

(a)

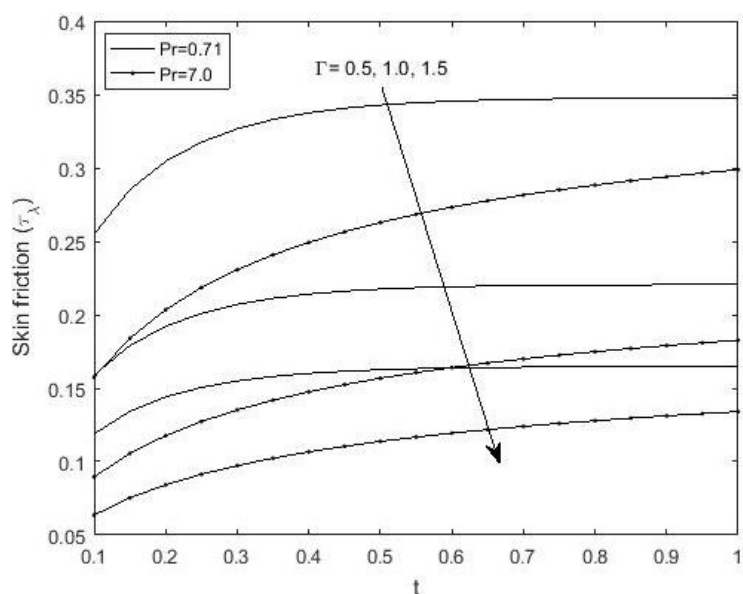

(b)

Figure 9. Variation of skin friction $\left(\tau_{\lambda}\right)$ for different values of $\Gamma(S=2.0, D a=0.1)$,

(a) Heat source, (b) Heat sink 
Table 2. Numerical values of the transient state Nusselt number obtained using the Riemann-sum approximation method $(R=2, \lambda=2)$

\begin{tabular}{cccc}
\hline$T$ & $S$ & $P r=0.71$ & $P r=7.0$ \\
\hline 0.1 & -2.0 & 0.2079 & 1.2078 \\
& -1.0 & 0.4067 & 1.2748 \\
& 0.0 & 0.6147 & 1.3422 \\
& 1.0 & 0.8328 & 1.4098 \\
& 2.0 & 1.0619 & 1.4778 \\
0.2 & -2.0 & 0.0016 & 0.8365 \\
& -1.0 & 0.2705 & 0.9306 \\
& 0.0 & 0.5689 & 1.0256 \\
& 1.0 & 0.8985 & 1.1215 \\
& 2.0 & 1.2653 & 1.2183 \\
0.3 & -2.0 & 0.1056 & 0.6554 \\
& -1.0 & 0.2224 & 0.7699 \\
& 0.0 & 0.6000 & 0.8861 \\
& 1.0 & 1.0422 & 1.0039 \\
& 2.0 & 1.5694 & 1.1234 \\
\hline
\end{tabular}

Table 3. Numerical values of the transient state mass flow rate obtained using the Riemann-sum approximation method $(\Gamma=1.0, D a=0.1, \lambda=$

\begin{tabular}{cccc}
\multicolumn{4}{c}{$2.0)$} \\
\hline$t$ & $S$ & $\operatorname{Pr}=0.71$ & $\operatorname{Pr}=7.0$ \\
\hline 0.1 & -2.0 & 27.3884 & 28.9306 \\
& -1.0 & 33.0275 & 29.9615 \\
& 0.0 & 38.8905 & 30.9986 \\
& 1.0 & 44.9358 & 32.0421 \\
& 2.0 & 51.0988 & 33.0920 \\
0.2 & -2.0 & 31.0949 & 31.1054 \\
& -1.0 & 35.8902 & 32.6510 \\
& 0.0 & 39.4276 & 34.2158 \\
& 1.0 & 40.5573 & 35.8003 \\
& 2.0 & 37.4492 & 37.4048 \\
0.3 & -2.0 & 30.3798 & 33.6425 \\
& -1.0 & 31.7712 & 35.6374 \\
& 0.0 & 27.8025 & 37.6703 \\
& 1.0 & 13.3778 & 39.7425 \\
& 2.0 & -20.6518 & 41.8550 \\
\hline
\end{tabular}

\section{CONCLUSION}

A theoretical analysis on the effect of heat source/sink on unsteady free convective flow in a coaxial cylinder saturated with porous material was carried out. The solutions of the governing momentum and energy equations where derived in the Laplace domain by applying the Laplace transform technique. The Laplace domain solution is then transformed to time domain using a numerical inversion scheme known as Riemann-sum approximation (RSA). For accuracy check, the result obtained using the Riemann-sum approximation (RSA) approach is validated by presenting a comparison with the steady state solution at large time. The main findings of this work are:

a. The fluid temperature is an increasing function of time, although the increase is more pronounced when heat source is applied.

b. Velocity increases with increase in Darcy number and time. The reverse trend is observed with increase in the viscosity ratio.

c. Skin frictions on both walls of the cylinder are enhanced with increase in Darcy number as time passes and drops as the viscosity ratio is increased.

\section{REFERENCES}

[1] B. K. Jha and T. S. Yusuf, "Transient free convective flow with heat generation/absorption in an annular porous medium: a semi-analytical approach,” J. Process Mech. Eng., vol. 232, no. 5, pp. 599-612, 2017.

[2] T. S. Yusuf and B. K. Jha, "A semi-analytical solution for time dependent natural convection flow with heat generation/absorption in an annulus partially filled with porous material," Multidiscip. Model. Mater. Struct., vol. 14, no. 5, pp. 1042-1063, 2018.

[3] T. S. Yusuf, "Exact solution of an MHD natural convection flow in vertical concentric annulus with heat absorption," Int. J. Fluid Mech. Thermal Sci., vol. 3, no. 5, pp. 52-61. 2017.

[4] M. A. Al-Nimr and T. T. Darabseh, "Analytical solution for transient laminar fully developed free convection in open-ended vertical concentric porous annuli," J. Heat Transf., vol. 117, no. 3, pp. 762-764, 1995.

[5] T. Paul and A. K. Singh, "Natural convection between coaxial vertical cylinders partially filled with a porous material," Forsch. Ing.-Wes., vol. 64, pp. 157-162, 1998.

[6] M. O. Oni, "Combined effect of heat source, porosity and thermal radiation on mixed convection flow in a vertical annulus: An exact solution," Eng. Sci. Tech., Int. J., vol. 20, no. 2, pp, 518-527, 2017.

[7] O. D. Makinde, Z. H. Khan, R. Ahmad, Rizwan Ul Haq, and W. A. Khan, "Unsteady MHD flow in a porous channel with thermal radiation and heat source/sink," International Journal of Applied and Computational Mathematics, vol. 5, no. 59, pp. 1-21, 2019.

[8] B. K. Jha and T. S. Yusuf, "Investigation of heat generation/absorption on natural convection flow in a vertical annular micro-channel: an exact solution," Multidiscip. Model. Mater. Struct., vol. 14, no. 5, pp. 143-167, 2018.

[9] B. K. Jha, M. O. Oni, and B. Aina, "Steady fully developed mixed convection flow in a vertical micro-concentric annulus with heat generating/absorbing fluid: an exact solution," Ain Shams Eng. J., vol. 9, no. 4, pp. 1289-1301, 2018.

[10] Y. Mahmoudi, "Constant wall heat flux boundary condition in micro-channels filled with a porous medium with internal heat generation under local thermal non-equilibrium condition," Int. J. Heat Mass Transf., vol. 85, pp. 524-542, 2015.

[11] S. Ostrach, "Combined natural and forced-convection flow and heat transfer of fluids with and without heat sources in channels with linearly varying wall temperatures," NACA TN 3141, 1954. 
[12] S. R. Mishraa, P. K. Pattnaikb, and G. C. Dash, "Effect of heat source and double stratification on MHD free convection in a micropolar fluid," Alexandria Engineering Journal, vol. 52, no. 3, pp. 681-689, 2015.

[13] R. M. Inman, "Experimental study of temperature distribution in laminar tube flow of a fluid with internal heat generation," Int. J. Heat Mass Transf., vol. 5, no. 11, pp. 1053-1054, 1962.

[14] S. Baag, S. R. Mishra, and B. K. Samantray, "Buoyancy effects on free convective MHD flow in the presence of heat source/sink," AMSE JOURNALS-AMSE IIETA. Modelling B; vol. 86, no. 1, pp 14-32, 2017.

[15] K. Bhattacharyya, "Effects of heat source/sink on MHD flow and heat transfer over a shrinking sheet with mass suction," Chem. Eng. Research Bulletin, vol. 15, no. 1, pp 12-17, 2011.

[16] D. Kumar and A. K. Singh, "Effects of heat source/sink and induced magnetic field on natural convective flow in vertical concentric annuli," Alexandria Eng. J., vol. 55, no. 4, pp. 3125-3133, 2016.

[17] A. O. Ajibade and J. J. Gambo, "Adomian decomposition method to magnetohydrodynamics natural convection heat generating/absorbing slip flow through a porous medium," Multidiscipline Modeling in Materials and Structures, vol. 15, no. 3, pp.673-684, 2019.

[18] M. R. Eid, "Chemical reaction effect on MHD boundary-layer flow of two-phase nanofluid model over an exponentially stretching sheet with a heat generation," J. Mol. Liq., vol. 220, pp. 718-725, 2016.

[19] M. R. Eid and K. L. Mahny, "Unsteady MHD heat and mass transfer of a non-Newtonian nanofluid flow of a two-phase model over a permeable stretching wall with heat generation/absorption," Adv. Powder Technol., vol. 28, no. 11, pp. 3063-3073, 2017.

[20] P. R. Sharma and G. Singh, "Effects of variable thermal conductivity and heat source/sink on MHD flow near a stagnation point on a linearly stretching sheet," J. Appl. Fluid Mech., vol. 2, no. 1, pp. 13-21, 2009.

[21] B. Lavanya and A. L. Ratnam, "Radiation and mass transfer effects on unsteady MHD natural convective flow past a vertical porous plate embedded in a porous medium in a slip flow regime with heat source/sink and Soret effect," Int. J. Eng. Tech. Res. vol. 2, no. 11, pp. 210-22, 2014.

[22] S. M. Ibrahim and K. Suneetha, "Effects of heat generation and thermal radiation on steady MHD flow near a stagnation point on a linear stretching sheet in porous medium and presence of variable thermal conductivity and mass transfer," J. Comput. Appl. Res. Mech. Eng. vol. 4, pp. 133-144, 2015.

[23] A. J. Chamkha, "Heat and mass transfer from MHD flow over a moving permeable cylinder with heat generation or absorption and chemical reaction," Commun. Numer. Anal., vol. 2011, pp. 1-20, 2011.

[24] D. Moalem, "Steady state heat transfer with porous medium with temperature dependent heat generation," Int. J. Heat Mass Transf., vol. 19, no. 5, pp. 529-537, 1976.

[25] O. Miyatake and T Fujii, "Free convection heat transfer between vertical plates - one plate isothermally heated and other thermally insulated," Heat Transf. Japan. Res., vol. 1, pp. 30-38, 1972.

[26] D. Y. Tzou, "Macro to Microscale Heat Transfer: The lagging behavior," Taylor and Francis, London, 1997.

\section{BIOGRAPHIES OF AUTHORS}

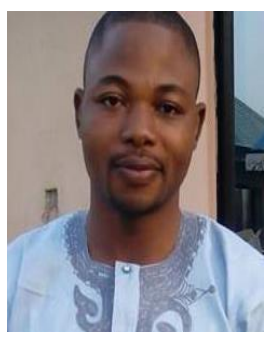

Taiwo S. Yusuf was born in Ikoyi, Kogi State, Nigeria. He is a lecturer and currently a PhD student in the Department of Mathematics Ahmadu Bello University (ABU), Zaria, Nigeria. He had his B.Sc. (first class honours) in the Department of Mathematics, ABU Zaria (2010). His areas of interest include flow through porous media, computational fluid dynamics, as well as heat and mass transfer.

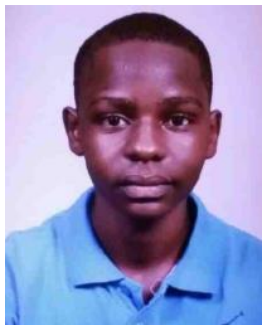

Gambo Dauda was born in Fika, Yobe State, Nigeria. He completed his bachelor degree from Ahmadu Bello University, Zaria, Nigeria. (first class honours) He is currently pursuing his MSc. in the field of applied mathematics at Ahmadu Bello University, Zaria, Nigeria. He has 2 years of research experience. Areas of expertise are computational fluid dynamics, fluid mechanics and heat and mass transfer. 